\title{
Identification of transcription factors at the site of implantation in the later stages of murine pregnancy
}

\author{
Baohui Zhao, Deanna Koon and Kathleen E Bethin \\ Wells Center for Pediatric Research, Department of Pediatrics, Indiana University School of Medicine, \\ James Whitcomb Riley Hospital for Children, 702 Barnhill Dr., RI 5960, Indianapolis, Indiana 46202, USA
}

Correspondence should be addressed to K E Bethin; Email kbethin@iupui.edu

\begin{abstract}
Despite medical advances, preterm delivery continues to complicate $12 \%$ of all births in the United States and is a major cause of neonatal deaths. One of the reasons that preterm labor continues to be a significant problem is that very little is understood about the factors involved in normal labor. Many investigators have studied parturition in the mouse and defined essential pathways for normal labor. Prostaglandins play an essential role in mouse labor and are important in human labor as well. We examined the 23 transcription factors from pregnant mouse uterus that change expression after the induction of cyclooxygenase-1, the enzyme that catalyzes the first committed step in prostaglandin synthesis. Using in situ hybridization, we have identified three of these transcription factors, Hoxa10, Hoxa11 and GILZ as being expressed in the decidua and regulated at the end of pregnancy. Both Hoxa10 and Hoxa11 are known to be critical for implantation, but very little is known about their roles in late gestation. GILZ has not previously been identified in the gravid uterus. In summary, we have identified three transcription factors that are regulated in the decidua at the end of pregnancy, suggesting a role in detachment of the fetus and placenta.

Reproduction (2006) 131 561-571
\end{abstract}

\section{Introduction}

Prematurely delivered infants account for $70-85 \%$ of all neonatal mortality and morbidity (American College of Obstetricians and Gynecologists 1995, Challis et al. 2000, Goldenberg et al. 2000, Smith et al. 2002). Survival of prematurely delivered infants has improved significantly in the last 20 years (Goldenberg 2002, Slattery \& Morrison 2002). However, the incidence of preterm labor has increased $27 \%$ since 1980 . In 2002, $12.1 \%$ of all infants born in the United States were premature (Martin et al. 2003). While intrauterine infection, multiple fetuses and fetal or uterine anomalies can account for some of these prematurely delivered infants, the etiology of the other $30-50 \%$ of preterm labor is unknown (American College of Obstetricians and Gynecologists 1995, Challis et al. 2000, Slattery \& Morrison 2002). This is due to the fact that we have only limited understanding of the processes involved in initiating normal labor. One of the hindrances in studying human labor is that many of the hormonal changes that occur during pregnancy are species specific (Smith et al. 2002). However, there are many factors involved in labor that are conserved from species to species (Lopez Bernal 2003, Elovitz \& Mrinalini, 2004, Jenkin \& Young 2004). By focusing on these similarities, studies in pregnant mice, sheep and other mammalian species have identified important pathways involved in parturition (Challis et al. 2000, Muglia 2000, Wang \& Hirsch 2003).

Because mouse gestation is short (19.5 days) and the mouse genome easy to manipulate, we use the mouse to study parturition. Murine genes that have been inactivated in the prostaglandin synthesis pathway have demonstrated that prostaglandins are essential for initiation of labor in the mouse (Sugimoto et al. 1997, Uozumi et al. 1997, Gross et al. 1998). In humans, prostaglandins are used to augment labor, and prostaglandin synthesis inhibitors are used to delay delivery, implicating an important role for prostaglandins in human labor as well (Novy \& Liggins 1980, Challis et al. 1997, 2002, Weiss 2000, Goldenberg 2002). Because of the importance of prostaglandins in both murine and human labor, we have focused on identifying genes in the mouse uterus that are affected by the induction of cyclooxygenase-1 (COX-1), which catalyzes the first committed step in prostaglandin synthesis.

In the pregnant wildtype mouse, COX-1 mRNA levels increase between gestational days 14.5 and 15.5 (Gross et al. 1998, Gross et al. 2000). Deficiency of COX-1 in the mouse results in delayed delivery of its pups. Because the induction of COX-1 is essential for normal 
labor, we previously used oligonucleotide microarray analysis to identify 504 uterine genes that change during murine pregnancy at least 2 -fold on gestational days 16.5 or 19.0 after the induction of COX-1 compared with day 13.5, which is before the induction of COX-1 (Bethin et al. 2003). Of these 504 genes, 23 encode for transcription factors. Since we hypothesized that many of the changes in uterine gene expression that take place during pregnancy are likely to be initiated by transcription factors, we analyzed the expression of all of these transcription factors by in situ hybridization. Only three of these transcription factors, Hoxa10, Hoxa11 and GILZ (glucocorticoidinduced leucine zipper) showed significant expression primarily in the implantation sites.

We are interested in the implantation site or decidua since it is the maternal surface that is in closest contact with the placenta and fetus and thus transmits signals from the fetus to the mother. In some species the fetus clearly plays an important role in the timing of parturition. Specifically, in the pregnant ewe, activation of the fetal hypothalamic-pituitary-adrenal (HPA) axis is critical for the normal onset of labor (McDonald \& Nathanielsz 1991, Jacobs et al. 1994, Challis et al. 2002, Lopez Bernal 2003). Although in humans, activation of the fetal HPA axis does not control the timing of parturition, it appears to play a supportive role in the timing of labor (Lopez Bernal 2003). Specifically, placental corticotropinreleasing hormone stimulates the fetal adrenals, which results in production of corticosteroids and dehydroepiandrosterone which stimulate fetal lung maturity and production of placental estrogens respectively (McLean et al. 1995, Majzoub et al. 1999, Weiss 2000, McGrath et al. 2002, Ni et al. 2004). We have postulated that signals from the fetus are likely to transmit changes in the uterus to prepare for parturition by affecting transcription factors. Because of the importance of the decidua in pregnancy, we have focused our work on the three transcription factors, Hoxa10, Hoxa11 and GILZ, that are regulated in the decidua near the end of pregnancy. In the present study, we show the in situ hybridization and Northern analysis of these three genes from the gravid mouse uterus.

Hoxa10 and Hoxa11 are homeobox genes that are essential for embryonic development (Krumlauf 1994) and are important for normal development of the mouse uterus (Hsieh-Li et al. 1995, Benson et al. 1996, Branford et al. 2000). Studies in knockout mice demonstrate that both transcription factors are essential for uterine receptivity and implantation (Hsieh-Li et al. 1995, Satokata et al. 1995, Benson et al. 1996, Gendron et al. 1997, Bagot et al. 2000). The location and regulation of Hoxa10 and Hoxa11 in humans is consistent with a similar role in human implantation as well (Taylor et al. 1998, 1999a, 1999b, Sakkas et al. 2003). In humans, Hoxa10 and Hoxa11 expression has been shown to persist in the decidua during the first trimester of pregnancy, but we were unable to find a reference detecting Hoxa11 in the decidua after the first trimester of pregnancy (Taylor et al.
1998, 1999a). High expression of Hoxa10 has been detected in term human pregnancy in the endometrium and fallopian tubes, and throughout pregnancy in the decidua and placenta (Evans et al. 2004, Salih \& Taylor 2004). Regulation of Hoxa10 in the decidua has also been shown in early, mid and late gestation of the pregnant baboon (Kim et al. 2003). The persistence of Hoxa10 expression in the decidua throughout pregnancy in humans, mice and baboons is highly suggestive of a conserved role in maintaining pregnancy. Likewise, the persistence of Hoxa11 expression in murine and human decidua suggests a role for Hoxa11 as well in maintaining pregnancy. Since Hoxa10 and Hoxa11 both appear to play important roles in murine and human implantation, and their expression persists in the decidua during pregnancy in both species, we postulated that any role discovered in murine parturition would help elucidate their roles in human parturition.

Three transcription factors, Hoxa10, Hoxa11 and GILZ, which are regulated in the decidua of the gravid mouse uterus are presented in this work. Here, we show by Northern analysis that in the gravid uterus expression of these three genes is down-regulated on gestational day 19.0, and we show by in situ hybridization that expression is primarily in the decidua. Although several authors have demonstrated Hoxa10 expression in the decidua of late pregnancy in humans and baboons, we could not find references to Hoxa10 and Hoxa11 expression in the mouse decidua in late pregnancy. To the best of our knowledge, this is the first study to demonstrate that GILZ is present in the gravid mouse uterus.

\section{Materials and Methods}

\section{Animal husbandry and tissue harvest}

All mouse protocols were in accordance with National Institutes of Health guidelines and approved by the Animal Care and Use Committee of Indiana University School of Medicine (Indianapolis, IN, USA). All mice used were 8-16 weeks old and of a C57BL/6 genetic background. Mice were housed on a $12 \mathrm{~h}$ light: $12 \mathrm{~h}$ darkness cycle with free access to rodent chow and water. Mating of estrous females to stud males was confirmed via detection of a copulation plug, with the morning of plug detection designated as day 0.5 of gestation. Plugged females were removed from the male cage to ensure accurate gestation timing.

Non-gravid, gestational day 13.5, 16.5 and 19.0 mice were killed by $\mathrm{CO}_{2}$ inhalation, and then the uteri were quickly isolated ( $n=4$ mice for gravid timepoints, $n=2$ or 3 for non-gravid samples). For gravid samples, the fetuses were removed, and the uteri isolated and rinsed in PBS. Half of the uterus was immediately frozen in liquid nitrogen and stored at $-80^{\circ} \mathrm{C}$ for total RNA isolation and half was fixed by immersion in $4 \%$ paraformaldehyde at $4^{\circ} \mathrm{C}$ for $24 \mathrm{~h}$. 


\section{In situ hybridization}

The paraformaldehyde-fixed uteri were cryopreserved in $10 \%$ sucrose in PBS and then embedded in OCT compound (Sakura Finetek, Torrance, CA, USA) for sectioning on a cryostat. Sixteen-micrometer cryostat sections through the uteri were collected and mounted on Superfrost plus slides. The slides were vacuum dried overnight and stored at $-80^{\circ} \mathrm{C}$.

In situ hybridization was carried out using modification of the Simmons et al. (1989) procedure. All templates were linearized from mouse expressed sequence tag (EST) clones (ATCC, Manassas, VA, USA) (IMAGE \# 6511608, 1958427, 476319 for Hoxa10, Hoxa11 and GILZ respectively). Hoxa10 and GILZ were linearized with EcoRI. Hoxa11 was linearized with Xho I. RNA probes were prepared with $\left[\alpha-{ }^{33} \mathrm{P}\right]$ UTP (MP Biomedicals, Irvine, CA, USA) by incubating $2 \mu \mathrm{g}$ of the linearized template with the appropriate polymerase as previously described (Bethin et al. 2000). T7 polymerase was used for Hoxa10 (GenBank accession number L08757). T3 polymerase was used for Hoxa11 and GILZ (GenBank accession numbers U20371 and AA050733 respectively). The approximate insert size was 2.6, 1.0 and $2.0 \mathrm{~kb}$ for Hoxa10, Hoxa11 and GILZ respectively. Uterine sections were pretreated with $10 \mu \mathrm{g} / \mathrm{ml}$ proteinase $\mathrm{K}$ for $30 \mathrm{~min}$ at $37^{\circ} \mathrm{C}$, blocked in $0.25 \%$ acetic anhydride for $10 \mathrm{~min}$, followed by dehydration in 50, 70, 95\% and absolute alcohol. Vacuumdried slides were hybridized to the appropriate antisense riboprobes in a humidified chamber for $16-20 \mathrm{~h}$ at $60-$ $65^{\circ} \mathrm{C}$. After washes and vacuum drying, slides were exposed to Biomax MR autoradiographic film (Eastman Kodak, Rochester, NY, USA) at room temperature for 410 days. Slides were subsequently dipped in 1:2 diluted Kodak NBT-2 or NBT emulsion, exposed in light-tight boxes at $4{ }^{\circ} \mathrm{C}$, and developed after determining proper exposure time from the films. Hoxa10 slides were exposed for 10 days, Hoxa11 for 4 days and GILZ for 3 days. Slides were counterstained with hematoxylin and eosin. All sense probes were generated from the same EST clones used for the antisense probes. All sense templates were linearized with Not I. Hoxa11 and GILZ sense templates were transcribed with T7 polymerase. Hoxa10 sense template was transcribed with SP6 polymerase. The sense probes were incubated under the identical conditions and the emulsion was exposed for the identical length of time as the corresponding antisense probes.

\section{Northern analysis}

RNA was prepared using Trizol (Invitrogen, Carlsbad, CA, USA) following the manufacturer's instructions. Ten micrograms of total RNA from four mice for each gestational timepoint (days 13.5, 16.5 and 19.0) and three virgin adult mice were subjected to electrophoresis through $1.2 \%$ agarose-formaldehyde gels and transferred to nitrocellulose membranes. $\left[\alpha-{ }^{32}\right.$ P]UTP-labeled RNA probes specific for mouse Hoxa10, Hoxa11 or GILZ mRNAs were generated by transcription of the linearized EST clone with the appropriate polymerase, as described above, and then hybridized at $65-68^{\circ} \mathrm{C}$ for $16-20 \mathrm{~h}$ in $50 \%$ formamide-containing buffer. After being washed, the blots were exposed to BioMax MS film (Kodak). Specificity of the probes for each of the genes was confirmed by detection of a $2.6 \mathrm{~kb}$ transcript for Hoxa10, $2.2 \mathrm{~kb}$ transcript for Hoxa11 and a $2.0 \mathrm{~kb}$ transcript for GILZ. Each mRNA hybridization signal was corrected for loading and recovery by normalization to the intensity of cyclophilin A hybridization. The films were scanned into Photoshop using an Epson Perfection 3490 Photo scanner. The band intensities were quantitated using NIH Image J (Abramoff et al. 2004). Statistical analyses of the normalized hybridization signal intensities were by ANOVA, with significance defined as $P<0.05$.

\section{Results}

In the gravid mouse, we identified three genes, Hoxa10, Hoxa11 and GILZ, that are transcription factors, primarily expressed in the decidua and whose expression significantly changes in late pregnancy. Expression levels were verified by Northern analysis and localization was determined by in situ hybridization. Shown in Figs 1-3 are representative sections from non-gravid $(n=2)$ and gravid days $13.5,16.5$ and 19.0 murine uteri $(n=4)$. Northern analysis for each gene was performed on uterine RNA isolated from non-gravid $(n=3)$ and gravid days 13.5, 16.5 and $19.0(n=4)$ mice (see Fig. 5). In the gravid mouse uterus, expression of Hoxa10 is primarily in the implantation site. There is also some expression of Hoxa10 in the endometrium in the gravid day 13.5 uterus (Fig. 1). In the non-gravid uterus there is faint expression of Hoxa10 in the endometrial stroma (Fig. 1). Images at $\times 200$ (Fig. 4) show the expression of Hoxa10 to be primarily in the decidual cells. In order to quantitate gene expression changes in the mouse uterus on gestational days 13.5, 16.5 and 19.0, Northern analysis was performed (Fig. 5 and Table 1). Northern analysis of uterine Hoxa10 demonstrated a $46 \%$ decrease on day 19.0 compared with day $13.5(P=0.001)$ with no difference in expression between days 13.5 and 16.5. Similar to Hoxa10, Hoxa11 expression is predominantly in the decidual cells (Figs 2 and 4). Hoxa11 signal intensity in the decidua is similar on days 16.5 and 13.5 , but is significantly reduced on day 19.0. In agreement with work by others (Hsieh-Li et al. 1995), there is some Hoxa11 signal in the non-gravid endometrial stroma. Northern analysis of the gravid uterus showed a $70 \%$ decrease in Hoxa11 expression on day 19.0 compared with day $13.5(P=0.0004)$ and slight decrease $(16 \%, P=0.02)$ on day 16.5 compared with day 13.5 (Fig. 5 \& Table 1).

GILZ was detected primarily in the implantation sites in the decidual cells $(\times 200$ images, Fig 4) with the lowest 

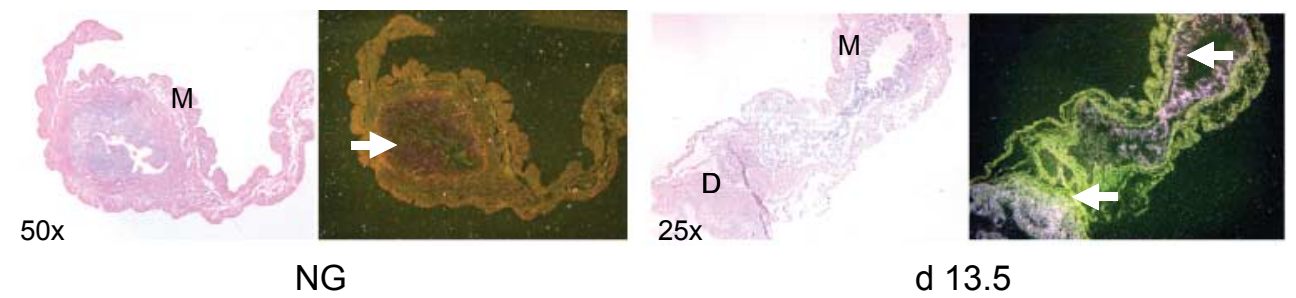

NG

d 13.5
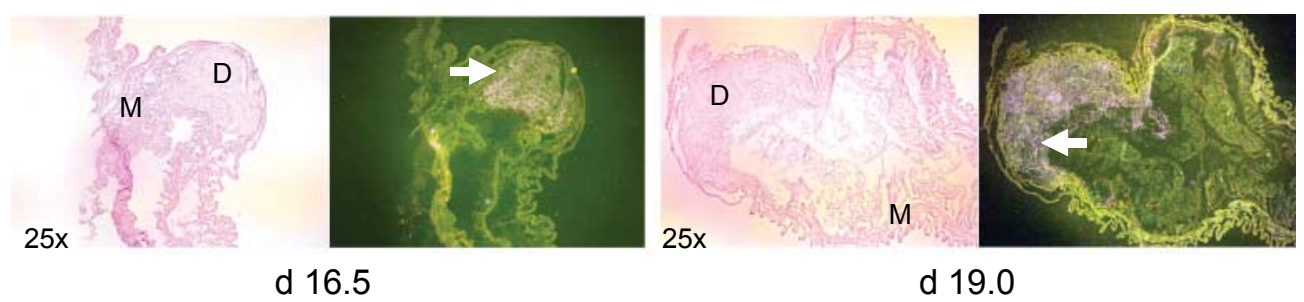

A

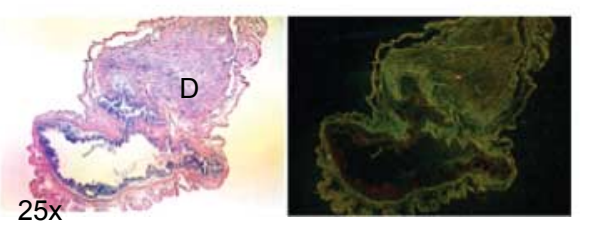

B

Figure 1 (A) In situ hybridization for Hoxa10 in the non-gravid and gravid mouse uterus. In situ hybridization was performed on four different mice from each gravid timepoint and two non-gravid mice. Shown are representative light-field images (on the left) and dark-field images (on the right) from murine non-gravid (NG) (magnification $\times 50$ ) or gravid day 13.5, 16.5 and 19.0 uteri (magnification $\times 25)$. The slides were counterstained with hematoxylin and eosin. Areas of hybridization appear white, demonstrating deposition of silver granules. The arrows are pointing to the hybridization signal. M, myometrium; D, decidua. (B) In situ hybridization using sense probe for Hoxa10. In situ hybridization using sense probe was performed on four different mice from gravid day 13.5 and 16.5 mice. Shown is a representative light-field image (on the left) and dark-field image (on the right) from a murine gravid day 13.5 uterus (magnification $\times 25$ ). The slides were counterstained with hematoxylin and eosin. No areas of hybridization are seen, demonstrated by a lack of silver granules. D, decidua.

expression detected in the day 19.0 uterus (Figs 3 and 5). On gestational day 19.0 there is strong GILZ expression in the endometrium, but very little expression in the decidua. In the non-gravid uterus GILZ expression is seen in the endometrial stroma. By Northern analysis, GILZ is decreased by $33 \%$ on day $19.0(P=0.002)$ and slightly increased $(114 \%, P=0.01)$ on day 16.5 compared with day 13.5 (Fig. 5 \& Table 1 ).

\section{Discussion}

Very little is understood about the factors involved in the initiation of normal labor or what goes wrong in idiopathic preterm labor. Although there is no animal model of labor that is identical to humans, some of the changes that occur during pregnancy are conserved across all mammalian species. Prostaglandin synthesis is one important factor in both human and murine labor. In an effort to understand what genes are important during labor, we identified by microarray analysis the genes that change in the mouse uterus after the induction of COX-1, which catalyzes the first committed step in prostaglandin synthesis. This work demonstrates that the transcription factors Hoxa10, Hoxa11 and GILZ are present at the sites of implantation in the mouse uterus near the end of pregnancy and that expression of these transcription factors changes after induction of COX-1. The location and regulation of these genes at the end of pregnancy suggest a role for these transcription factors in the parturition process. Here, we postulate the roles these genes may be playing in parturition based on what is known about these transcription factors.

In the adult mouse, Hoxa10 is expressed throughout the uterus, with the highest expression in the endometrium (Troy et al. 2003). In normal mouse pregnancy, Hoxa10 expression can be seen on days 0.5 and 1.5 post-conception in the endometrium (Satokata et al. 1995). This expression shifts to the endometrial stroma on day 3.5 and to the decidua on day 5.5. Expression peaks between days 3.5 and 4.5 and is down-regulated between days 4.5 and 7.5 (Satokata et al. 1995). Expression at day 7.5 in the gravid mouse uterus is the latest that we could find. Parturition in the Hoxa10-deficient mouse has not been studied since the pregnancies abort at the time of implantation.

In normal mouse pregnancy, Hoxa11 expression is first detected on gestational day 3 in the uterine stroma. Hoxa11 expression has been shown to peak between gestational days 6 and 8 in the decidua (Gendron et al. 1997). To the best of our knowledge, this work represents 

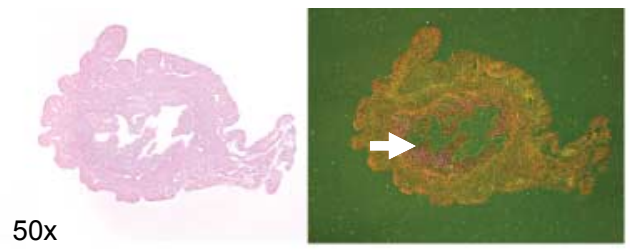

NG

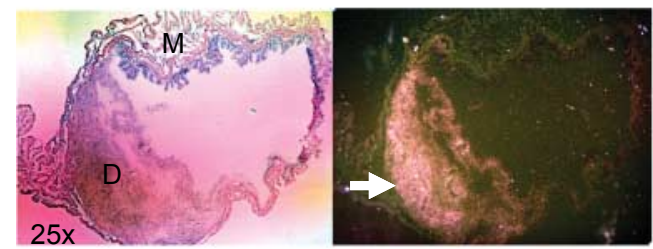

d 16.5

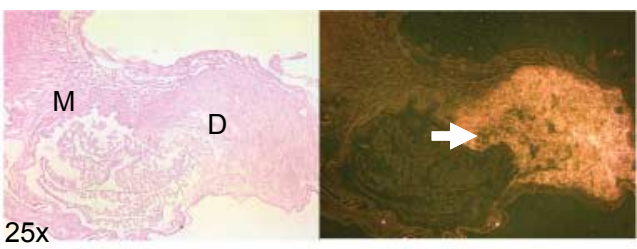

d 13.5

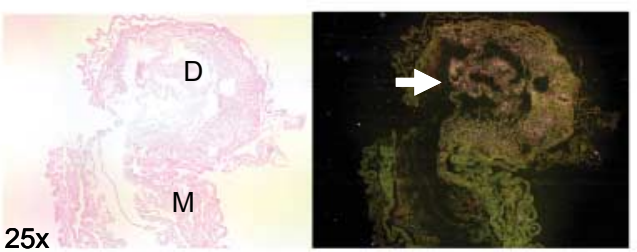

d 19.0

A

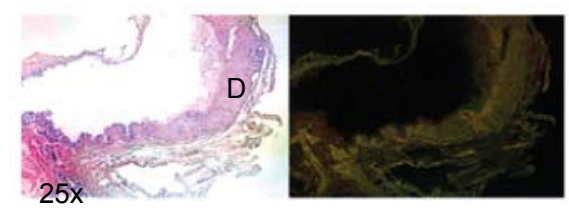

B

Figure 2 (A) In situ hybridization for Hoxa11 in the non-gravid and gravid mouse uterus. In situ hybridization was performed on four different mice from each gravid timepoint and two non-gravid mice. Shown is a representative light-field images (on the left) and dark-field images (on the right) from murine non-gravid (NG) (magnification $\times 50$ ) or gravid day 13.5, 16.5 and 19.0 uteri (magnification $\times 25$ ). The slides were counterstained with hematoxylin and eosin. Areas of hybridization appear white, demonstrating deposition of silver granules. The arrows are pointing to the hybridization signal. M, myometrium; D, decidua. (B) In situ hybridization using sense probe for Hoxa11. In situ hybridization using sense probe was performed on four different mice from gravid days 13.5 and 16.5. Shown is a representative light-field image (on the left) and dark-field image (on the right) from a murine gravid day 13.5 uterus (magnification $\times 25$ ). The slides were counterstained with hematoxylin and eosin. No areas of hybridization are seen, demonstrated by a lack of silver granules. D, decidua.

the latest in mouse gestation that Hoxa11 expression has been examined. The Hoxa11 knockout mouse is sterile and the heterozygote mouse has a markedly reduced litter size (Hsieh-Li et al. 1995). Since the Hoxa11-null females fail to undergo a normal decidual cell reaction in response to intraluminal injection of oil in pseudopregnant females, it appears that there is a defect in decidualization, which is critical for implantation (Gendron et al. 1997). Similar to the Hoxa10-deficient mouse, parturition can not be studied in the Hoxa11-deficient mouse as the embryos do not implant.

Our results show that both Hoxa10 and Hoxa11 are highly expressed in the implantation sites of pregnant mice long after implantation is completed. Studies in ovariectomized mice show that administration of progesterone results in an increase in uterine expression of both Hoxa10 and Hoxa11 RNA. Similarly, coadministration of the progesterone antagonist, RU486 blocks the progesterone-stimulated increase in both Hoxa10 and Hoxa11 (Ma et al. 1998). In murine pregnancy, serum progesterone falls 1-2 days prior to delivery. Based on the responsiveness of Hoxa10 and Hoxa11 expression to progesterone, one would predict that these genes would decrease in expression near the end of pregnancy. Indeed, both the Northern and in situ hybridization data show that the expression of Hoxa10 and Hoxa11 are decreased in the gestational day 19.0 uterus compared with day 13.5. Based on expression in the decidua, decreased expression at the end of pregnancy and their important role in implantation, we have postulated that Hoxa10 and Hoxa11 may play a role in regulating genes involved in detachment of the embryo and placenta during parturition.

In humans, Hoxa10 and Hoxa11 expression in the uterus has been studied extensively. Hoxa10 RNA is detected in the human endometrium throughout the menstrual cycle, but increases during the mid-secretory phase when estrogen and progesterone are both present. (Taylor et al. 1998, Cermik et al. 2001). In cultured endometrial stromal cells Hoxa10 expression was increased by the addition of estrogen, progesterone or both. However, in women with endometriosis, Hoxa10 levels in the endometrium fail to increase during the mid-secretory phase of the menstrual cycle (Taylor et al. 1999b). This abnormal expression of Hoxa10 in women with endometriosis may contribute to the infertility associated with endometriosis. Polycystic ovary syndrome (PCOS) is a condition in women associated with anovulatory cycles, hyperandrogenism and insulin resistance. Even after ovulation is corrected in women with PCOS, fertility rates are very low. 


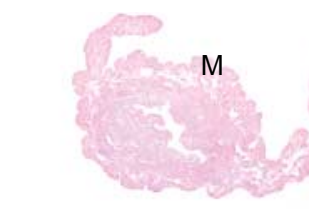

$50 x$



NG

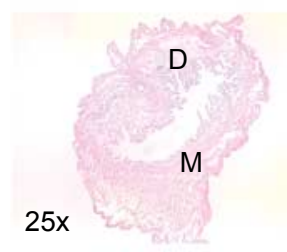

A

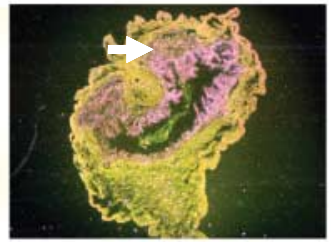

d 16.5

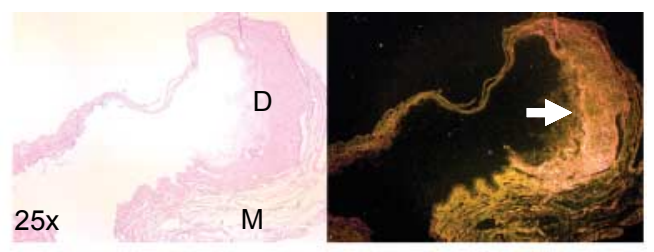

d 13.5

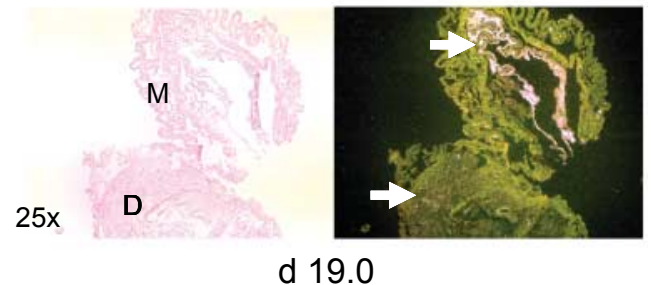

d 19.0

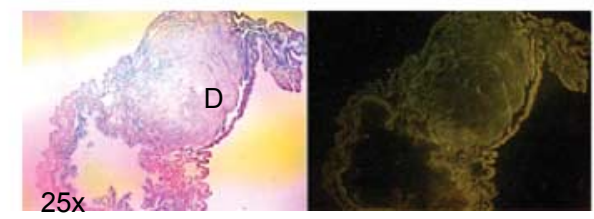

B

Figure 3 (A) In situ hybridization for GILZ in the non-gravid and gravid mouse uterus. In situ hybridization was performed on four different mice from each gravid timepoint and two non-gravid mice. Shown are representative light-field images (on the left) and dark-field images (on the right) from murine non-gravid (NG) (magnification $\times 50$ ) or gravid day 13.5, 16.0 and 19.0 uteri (magnification $\times 25)$. The slides were counterstained with hematoxylin and eosin. Areas of hybridization appear white, demonstrating deposition of silver granules. The arrows are pointing to the hybridization signal. M, myometrium; D, decidua. (B) In situ hybridization using sense probe for GILZ. In situ hybridization using sense probe was performed on four different mice from gravid day 13.5 and 16.5. Shown is a representative light-field image (on the left) and darkfield image (on the right) from a murine gravid day 13.5 uterus (magnification $\times 25$ ). The slides were counterstained with hematoxylin and eosin. No areas of hybridization are seen, demonstrated by a lack of silver granules. D, decidua.

Hoxa10 levels are also reduced in the endometrium during the mid-secretory phase of women with PCOS during ovulatory cycles (Eun Kwon \& Taylor 2004). When Ishikawa cells, an endometrial adenocarcinoma cell line, are grown in conditioned media from preimplantation human embryos, Hoxa10 expression is induced (Sakkas et al. 2003). The steroid and menstrual cycle regulation of Hoxa10 expression, the abnormal expression of Hoxa10 in women with endometriosis and PCOS and the ability of conditioned media from preimplantation blastocysts to induce Hoxa10 expression all implicate Hoxa10 as a likely player in human implantation.

Hoxa11 is also expressed in the human endometrium throughout the menstrual cycle and increases during the mid-secretory phase, which occurs at the time of implantation (Taylor et al. 1999a, Wang et al. 2004). Similar to Hoxa10, Hoxa11 expression increases in cultured endometrial stromal cells in response to estrogen, progesterone or both. And, in women with endometriosis, Hoxa11 levels also fail to increase during the midsecretory phase of the menstrual cycle. Although the pathogenesis of infertility in women with endometriosis is unclear, abnormal expression of Hoxa10 and Hoxa11 is consistent with a role for both in implantation and the infertility associated with endometriosis (Taylor et al. 1999b).

Significant expression of both Hoxa10 and Hoxa11 persists in the decidua of women at least during the first trimester of pregnancy, suggesting a role for both in the maintenance of pregnancy as well as a role in implantation (Taylor et al. 1998, 1999a). Hoxa10 expression in women has also been detected in late pregnancy in the endometrium and in the decidua and placenta during all three trimesters (Evans et al. 2004, Salih \& Taylor 2004). Similarly to in mice, decidual expression of Hoxa10 in the pregnant baboon was decreased in term pregnancy compared with endometrium from early pregnancy (Kim et al. 2003). However, in humans, Hoxa10 expression in the decidua was highest in the third trimester of pregnancy (Evans et al. 2004). These third-trimester decidual samples were from women who were undergoing elective cesarean sections and their labor status was not reported. It is possible that the difference between the human and murine samples is that the mice were in labor and the women were not. Since Hoxa10 and Hoxa11 appear to play a similar role in mice and humans during implantation and are expressed in both in the decidua after implantation is completed, we have postulated that their role at the 


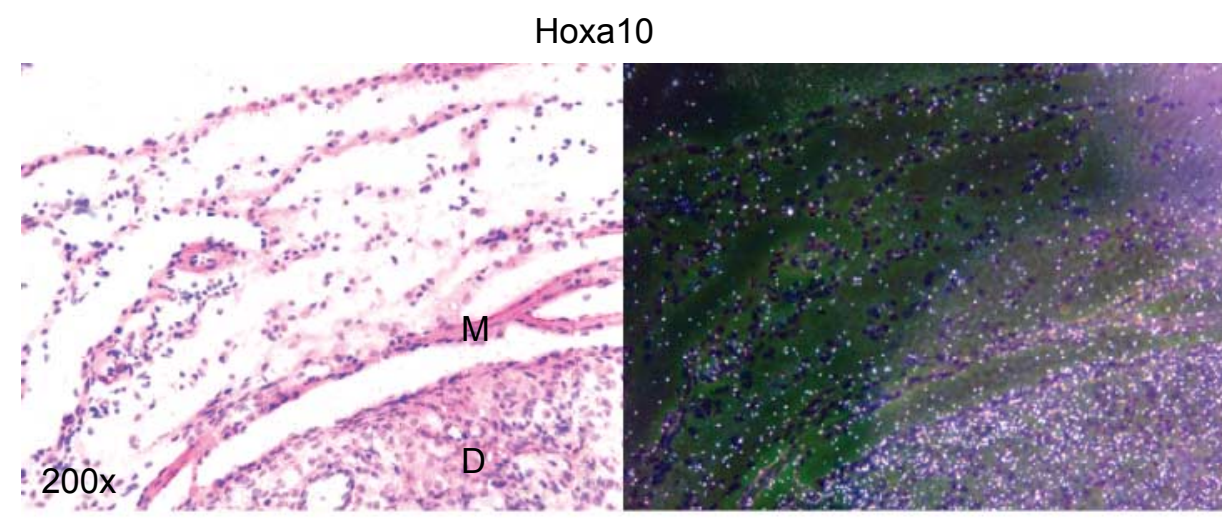

Hoxa11

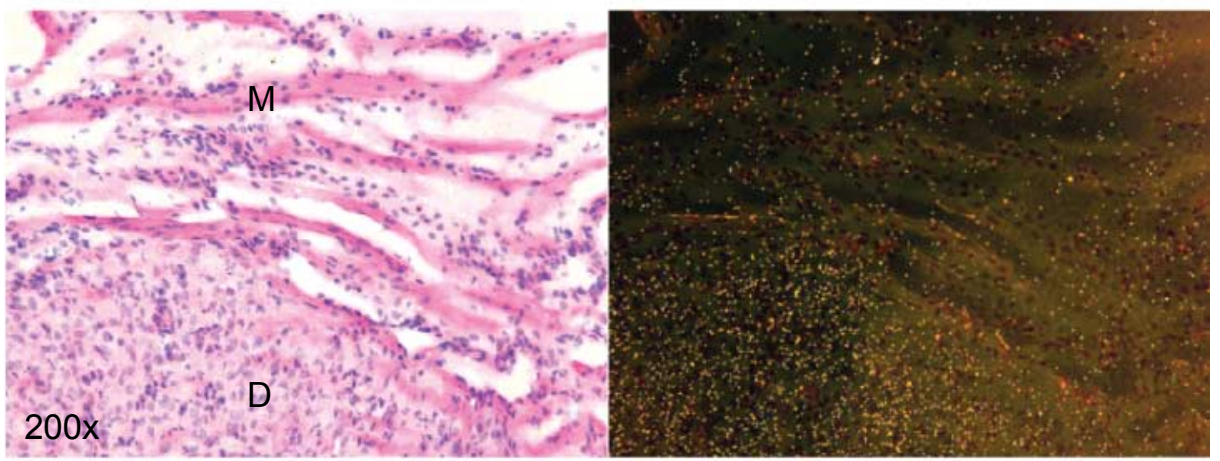

GILZ

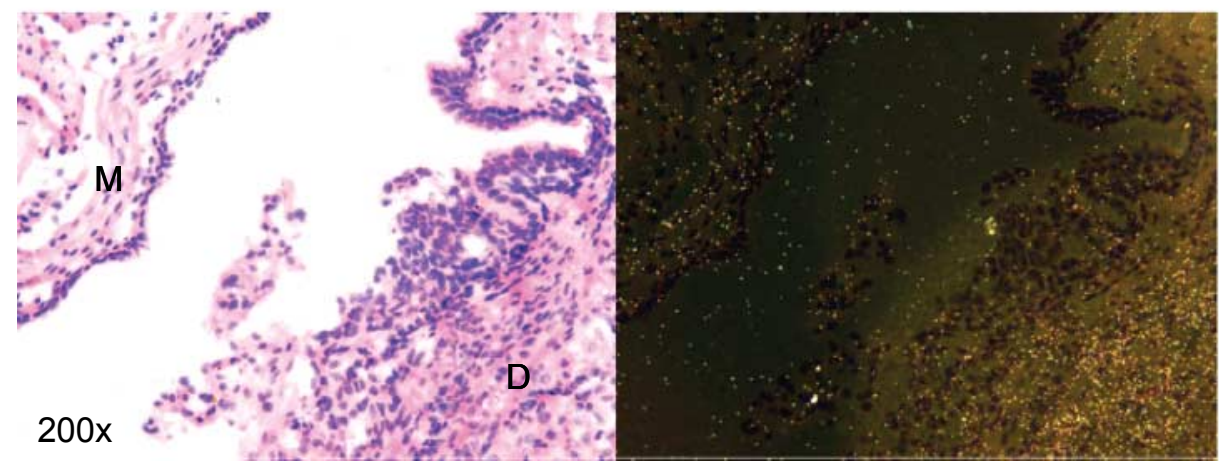

Figure 4 Gravid uterine expression of Hoxa10, Hoxa11 and GILZ at $\times 200$ magnification. The in situ hybridization slides from Figs $1-3$ were viewed under $\times 200$ magnification. Shown are representative decidua from a gravid day 13.5 mouse uterus hybridized to antisense probe for Hoxa10 or Hoxa11 or a gravid day 16.5 uterus probed for GILZ. Shown are representative light-field images (on the left) and dark-field images (on the right). The slides were counterstained with hematoxylin and eosin. Areas of hybridization appear white, demonstrating deposition of silver granules. end of pregnancy in mice will help elucidate their role in humans.

Hoxa10 is also expressed in human myometrium throughout the menstrual cycle, but is maximal in the proliferative and early secretory phases of the menstrual cycle, when progesterone is low. Experiments with myometrial cell culture confirmed that Hoxa10 expression is decreased in the myometrium in response to progesterone (Cermik et al. 2001). Hoxa10 has also been detected in the myometrium of pregnant women at term, but the relationship of Hoxa10 levels with labor is controversial (Mesiano et al. 2002, Esplin et al. 2005). These data suggest that Hoxa10 in the myometrium and decidua are regulated differently.

GILZ was first identified in the mouse thymus as a dexamethasone-responsive gene (D'Adamio et al. 1997,
Ayroldi et al. 2002). GILZ shares significant amino acid sequence identity with TSC-22 (TGF $\beta$-Stimulated Clone) and delta sleep inducing peptide immunoreactor (DSIPI) (Vogel et al. 1996, Tynan et al. 2004). GILZ has been shown to be a glucocorticoid-induced gene in human lymphohematopoietic cells (Cannarile et al. 2001). GILZ modulates T-lymphocyte activation and apoptosis ( $\mathrm{D}^{\prime}$ Adamio et al. 1997, Riccardi et al. 1999). It is highly expressed in B cells and down-regulated in activated B cells (Kester et al. 1999, Glynne et al. 2000). It has been postulated that down-regulation of GILZ facilitates B-cell activation (Glynne et al. 2000). Expression of GILZ, TSC22 and DSIPI has been studied in several murine and human tissues (Vogel et al. 1996, D'Adamio et al. 1997, Cannarile et al. 2001). However, we were not able to find literature demonstrating expression of any of these genes 

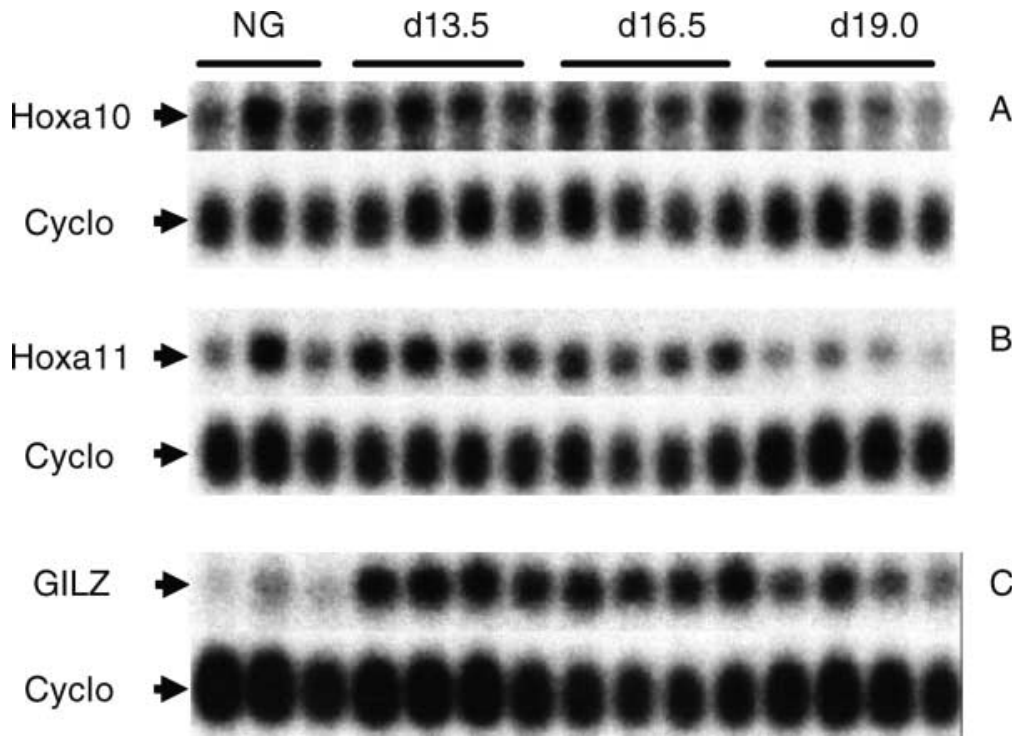

A

B

Figure 5 Northern blots of Hoxa10, Hoxa11 and GILZ in the non-gravid and gravid mouse uterus. Ten micrograms of total RNA from four mice for each gestational timepoint (days 13.5, 16.5 and 19.0) and three virgin adult mice were subjected to electrophoresis. Specificity of the probes for each of the genes was confirmed by detection of a $2.6 \mathrm{~kb}$ transcript for Hoxa10, $2.2 \mathrm{~kb}$ transcript for Hoxa11 and a $2.0 \mathrm{~kb}$ transcript for GILZ. Each mRNA hybridization signal was corrected for loading and recovery by normalization to intensity of cyclophilin A hybridization. in the uterus. The GILZ promoter has been shown to have an estrogen response element. Interestingly, estrogen down-regulates GILZ in MCF-7 breast adenocarcinoma cells, but up-regulates GILZ in HEK293 and HeLa cells (Tynan et al. 2004). Since estrogen increases near the end of murine pregnancy, it is plausible that estrogen downregulates GILZ in the decidua between gestational days 16.5 and 19.0 (Watanabe et al. 2003).

GILZ may also be playing a role in inducing COX-2 (cyclooxygenase 2) and prostaglandin synthesis during delivery. Not only is COX-1 essential for normal labor, but COX-2 also plays a role in normal parturition. During delivery, COX-1 is down-regulated and COX-2, located in the decidua and myometrium, is up-regulated (Tsuboi et al. 2000, 2003). COX-2 is known to be critical for the early stages of pregnancy (Lim et al. 1997). Since COX-2deficient mice abort early in pregnancy, the necessity for COX-2 in the later stages of pregnancy remains controversial (Reese et al. 2000, Tsuboi et al. 2003). GILZ has been shown to play a putative role in the anti-inflammatory and immunosuppressive effects of glucocorticoids by interacting with Raf-1 and inhibiting the classic MAPK (mitogen-activated protein kinase) pathway (Ayroldi et al. 2002). In the pregnant mouse, p38 MAPK activity is significantly up-regulated in the decidua and has been postulated to be involved in the increase in prostaglandin production from COX-2 in the decidua during active labor

Table 1 Average RNA levels \pm S.D. as determined by Image for Hoxa10, Hoxa11 and GILZ from gravid days 13.5, 16.5 and 19.0 mouse uteri.

\begin{tabular}{lccc}
\hline & Day $\mathbf{1 3 . 5}$ & Day $\mathbf{1 6 . 5}$ & Day $\mathbf{1 9 . 0}$ \\
\hline Hoxa10 & $10052 \pm 841$ & $11622 \pm 968$ & $5394 \pm 1396 *$ \\
Hoxa11 & $16338 \pm 810$ & $13784 \pm 284 *$ & $4891 \pm 702 *$ \\
GILZ & $20182 \pm 411$ & $23101 \pm 673 *$ & $13463 \pm 1219 *$ \\
\hline
\end{tabular}

$* P<0.05$ compared with day 13.5 .
(Takanami-Ohnishi et al. 2001). Furthermore, inhibition of p38 MAPK abolishes the increase in stromal COX-2 in response to a deciduogenic stimulus (Scherle et al. 2000). It remains to be determined whether down-regulation of GILZ in the decidua between gestational days 13.5 and 19.0 results in activation of the classic MAPK pathway, providing another mechanism for induction of COX-2 and prostaglandin production. There is no published knockout of GILZ, so its affects on parturition are unknown. We postulate that down-regulation of GILZ near the end of pregnancy activates the immune system and the MAPK pathway in the decidua, helping to facilitate detachment of the fetus/placenta from the uterus and stimulating uterine contractions.

Down-regulation of GILZ in the decidua may also play a role in the parturition cascade through its regulation of nuclear factor kappa B (NF-кB) (Lindstrom \& Bennett 2004). In vitro, GILZ co-immunoprecipitates with p65, which would inhibit transactivation of NF-кB (Lindstrom \& Bennett 2004). Release of GILZ's inhibition (by downregulation of GILZ) of NF-кB may play an important role in the parturition cascade. The murine and human COX-2 promoters contain a conserved pair of NF- $\mathrm{kB}$-binding sites (Lindstrom \& Bennett 2004). NF-кB activation of COX-2 has been shown in immortalized myometrial cells, WISH cells (immortalized amniotic cells) and in amniotic cells during labor (Wang \& Tai 1998, Belt et al. 1999, Allport et al. 2001). In vivo, stimulation of NF-кB is associated with a preterm delivery mouse model and injection of an inhibitor of NF-кB delays parturition (Condon et al. 2004). Therefore, down-regulation of GILZ in the decidua and its regulation of NF- $\mathrm{B}$ provides another mechanism for activation of COX-2 and prostaglandin synthesis during labor.

In summary, we have identified two transcription factors, Hoxa10 and Hoxa11, known to be important in implantation, whose expression is also regulated in the implantation site near the end of pregnancy. Up-regulation 
of Hoxa10 and Hoxa11 is essential for the decidualization reaction that allows the embryo to attach to the uterine wall during implantation. We postulate that down-regulation of these same transcription factors near the end of pregnancy helps facilitate the opposite reaction, detachment of the fetus and placenta from the uterus. We have also identified one other transcription factor, GILZ, that was not known to have any role in pregnancy, as also being regulated in the implantation site near the end of pregnancy. The role of GILZ in the immune system suggests its role in the implantation site is to activate the immune system near the end of pregnancy to facilitate detachment of the fetus and placenta. Further, its putative role in regulating the classic MAPK pathway and NF- $\mathrm{BB}$ may help to initiate uterine contractions at the site of implantation. Based on their locations and regulation at the end of murine pregnancy, Hoxa10, Hoxa11 and GILZ likely play a role in parturition. Creating a transgenic mouse that can be induced to overexpress these genes near the end of pregnancy or a conditional knockout will help define their role in parturition.

\section{Acknowledgements}

We wish to thank Allyson Curtis, Drs Louis Muglia, Ora Pescovitz, Rosemary Steinmetz and Robert Bigsby for helpful discussions and manuscript review. This work was supported by the Lawson Wilkins Pediatric Endocrine Society Abbott Clinical Scholars Award to K E B, grant K08-HD01483 from the $\mathrm{NIH}$ to KE B and the Riley Children's Foundation. The authors declare that there is no conflict of interest that would prejudice the impartiality of this scientific work.

\section{References}

Abramoff MD, Magelhaes PJ \& Ram SJ 2004 Image processing with ImageJ. Biophotonics International 11 36-42.

Allport VC, Pieber D, Slater DM, Newton R, White JO \& Bennett PR 2001 Human labour is associated with nuclear factor-kappaB activity which mediates cyclo-oxygenase-2 expression and is involved with the 'functional progesterone withdrawal'. Molecular Human Reproduction 7 581-586.

American College of Obstetricians and Gynecologists 1995 Preterm labor. ACOG Technical Bulletin 206 1-10.

Ayroldi E, Zollo O, Macchiarulo A, Di Marco B, Marchetti C \& Riccardi C 2002 Glucocorticoid-induced leucine zipper inhibits the Raf-extracellular signal-regulated kinase pathway by binding to Raf-1. Molecular and Cellular Biology 22 7929-7941.

Bagot CN, Troy PJ \& Taylor HS 2000 Alteration of maternal Hoxa10 expression by in vivo gene transfection affects implantation. Gene Therapy 7 1378-1384.

Belt AR, Baldassare JJ, Molnar M, Romero R \& Hertelendy F 1999 The nuclear transcription factor NF-kappaB mediates interleukin1 beta-induced expression of cyclooxygenase-2 in human myometrial cells. American Journal of Obstetrics and Gynecology 181 359-366.

Benson G, Lim H, Paria B, Satokata I, Dey S \& Maas R 1996 Mechanisms of reduced fertility in Hoxa-10 mutant mice: uterine homeosis and loss of maternal Hoxa-10 expression. Development 122 2687-2696.

Bethin KE, Vogt SK \& Muglia LJ 2000 Interleukin-6 is an essential, corticotropin-releasing hormone-independent stimulator of the adrenal axis during immune system activation. PNAS 97 9317-9322.

Bethin KE, Nagai Y, Sladek R, Asada M, Sadovsky Y, Hudson TJ \& Muglia LJ 2003 Microarray analysis of uterine gene expression in mouse and human pregnancy. Molecular Endocrinology 17 1454-1469.

Branford WW, Benson GV, Ma L, Maas RL \& Potter SS 2000 Characterization of Hoxa-10/Hoxa-11 transheterozygotes reveals functional redundancy and regulatory interactions. Developmental Biology 224 373-387.

Cannarile L, Zollo O, D'Adamio F, Ayroldi E, Marchetti C, Tabilio A, Bruscoli S \& Riccardi C 2001 Cloning, chromosomal assignment and tissue distribution of human GILZ, a glucocorticoid hormoneinduced gene. Cell Death and Differentiation 8 201-203.

Cermik D, Karaca M \& Taylor HS 2001 HOXA10 expression is repressed by progesterone in the myometrium: differential tissue-specific regulation of HOX gene expression in the reproductive tract. Journal of Clinical Endocrinology and Metabolism 86 3387-3392.

Challis JR, Lye SJ \& Gibb W 1997 Prostaglandins and parturition. Annals of the New York Academy of Sciences 828 254-267.

Challis JR, Sloboda DM, Alfaidy N, Lye SJ, Gibb W, Patel FA, Whittle WL \& Newnham JP 2002 Prostaglandins and mechanisms of preterm birth. Reproduction 124 1-17.

Challis JRG, Matthews SG, Gibb W \& Lye SJ 2000 Endocrine and paracrine regulation of birth at term and preterm. Endocrine Reviews 21 514-550.

Condon JC, Jeyasuria P, Faust JM \& Mendelson CR 2004 Surfactant protein secreted by the maturing mouse fetal lung acts as a hormone that signals the initiation of parturition. PNAS 101 4978-4983.

D'Adamio F, Zollo O, Moraca R, Ayroldi E, Bruscoli S, Bartoli A, Cannarile L, Migliorati G \& Riccardi C 1997 A new dexamethasone-induced gene of the leucine zipper family protects T lymphocytes from TCR/CD3-activated cell death. Immunity 7 803-812.

Elovitz MA \& Mrinalini C 2004 Animal models of preterm birth. Trends in Endocrinology and Metabolism 15 479-487.

Esplin MS, Fausett MB, Peltier MR, Hamblin S, Silver RM, Branch DW, Adashi EY \& Whiting D 2005 The use of cDNA microarray to identify differentially expressed labor-associated genes within the human myometrium during labor. American Journal of Obstetrics and Gynecology 193 404-413.

Eun Kwon H \& Taylor HS 2004 The role of HOX genes in human implantation. Annals of the New York Academy of Sciences 1034 $1-18$.

Evans KN, Bulmer JN, Kilby MD \& Hewison M 2004 Vitamin D and placental-decidual function. Journal of the Society for Gynecologic Investigation 11 263-271.

Gendron RL, Paradis H, Hsieh-Li HM, Lee DW, Potter SS \& Markoff E 1997 Abnormal uterine stromal and glandular function associated with maternal reproductive defects in Hoxa-11 null mice. Biology of Reproduction 56 1097-1105.

Glynne R, Ghandour G, Rayner J, Mack DH \& Goodnow CC 2000 B-lymphocyte quiescence, tolerance and activation as viewed by global gene expression profiling on microarrays. Immunological Reviews $176216-246$.

Goldenberg RL 2002 The management of preterm labor. Obstetrics and Gynecology 100 1020-1037.

Goldenberg RL, Hauth JC \& Andrews WW 2000 Intrauterine infection and preterm delivery. New England Journal of Medicine 342 1500-1507.

Gross G, Imamura T \& Muglia LJ 2000 Gene knockout mice in the study of parturition. Journal of the Society for Gynecologic Investigation 7 88-95.

Gross GA, Imamura T, Luedke CE, Vogt SK, Olson LM, Nelson DM, Sadovsky Y \& Muglia LJ 1998 Opposing actions of prostaglandins and oxytocin determine the onset of murine labor. PNAS 95 $11871-11875$.

Hsieh-Li H, Witte D, Weinstein M, Branford W, Li H, Small K \& Potter S 1995 Hoxa 11 structure, extensive antisense transcription, 
and function in male and female fertility. Development 121 1373-1385.

Jacobs RA, Young IR, Hollingworth SA \& Thorburn GD 1994 Chronic administration of low doses of adrenocorticotropin to hypophysectomized fetal sheep leads to normal term labor. Endocrinology 134 1389-1394.

Jenkin G \& Young IR 2004 Mechanisms responsible for parturition; the use of experimental models. Animal Reproduction Science 82-83 $567-581$.

Kester HA, Blanchetot C, den Hertog J, van der Saag PT \& van der Burg B 1999 Transforming growth factor-beta-stimulated clone-22 is a member of a family of leucine zipper proteins that can homoand heterodimerize and has transcriptional repressor activity. Journal of Biological Chemistry 274 27439-27447.

Kim JJ, Taylor HS, Akbas GE, Foucher I, Trembleau A, Jaffe RC, Fazleabas AT \& Unterman TG 2003 Regulation of insulin-like growth factor binding protein-1 promoter activity by FKHR and HOXA10 in primate endometrial cells. Biology of Reproduction 68 24-30.

Krumlauf R 1994 Hox genes in vertebrate development. Cell $\mathbf{7 8}$ 191-201.

Lim H, Paria BC, Das SK, Dinchuk JE, Langenbach R, Trzaskos M \& Dey SK 1997 Multiple fetal reproductive failures in cyclooxygenase-2-deficient mice. Cell 91 197-208.

Lindstrom T \& Bennett P 2004 Transcriptional regulation of genes for enzymes of the prostaglandin biosynthetic pathway. Prostaglandins, Leukotrienes, and Essential Fatty Acids 70 115-135.

Lopez Bernal A 2003 Mechanisms of labour-biochemical aspects. BJOG 110 (Supplement 20) 39-45.

Ma L, Benson GV, Lim H, Dey SK \& Maas RL 1998 Abdominal B $(\mathrm{AbdB})$ Hoxa genes: regulation in adult uterus by estrogen and progesterone and repression in mullerian duct by the synthetic estrogen diethylstilbestrol (DES). Developmental Biology 197 $141-154$.

Majzoub JA, McGregor JA, Lockwood CJ, Smith R, Taggart MS \& Schulkin J 1999 A central theory of preterm and term labor: putative role for corticotropin-releasing hormone. American Journal of Obstetrics and Gynecology 180 S232-S241.

Martin JA, Hamilton BE, Sutton PD, Ventura SJ, Menacker F \& Muson M 2003 Births: final data for 2002. National Vital Statistics Reports 52 1-113.

McDonald TJ \& Nathanielsz PW 1991 Bilateral destruction of the fetal paraventricular nuclei prolongs gestation in sheep. American Journal of Obstetrics and Gynecology 165 764-770.

McGrath S, McLean M, Smith D, Bisits A, Giles W \& Smith R 2002 Maternal plasma corticotropin-releasing hormone trajectories vary depending on the cause of preterm delivery. American Journal of Obstetrics and Gynecology 186 257-260.

McLean M, Bisits A, Davies J, Woods R, Lowry P \& Smith R 1995 A placental clock controlling the length of human pregnancy. Nature Medicine 1 460-463.

Mesiano S, Chan E-C, Fitter JT, Kwek K, Yeo G \& Smith R 2002 Progesterone withdrawal and estrogen activation in human parturition are coordinated by progesterone receptor A expression in the myometrium. Journal of Clinical Endocrinology and Metabolism 87 2924-2930.

Muglia LJ 2000 Genetic analysis of fetal development and parturition control in the mouse. Pediatric Research 47 437-443.

Ni X, Hou Y, Yang R, Tang X, Smith R \& Nicholson RC 2004 Progesterone receptors $\mathrm{A}$ and $\mathrm{B}$ differentially modulate corticotropin-releasing hormone gene expression through a cAMP regulatory element. Cellular and Molecular Life Sciences 61 1114-1122.

Novy MJ \& Liggins GC 1980 Role of prostaglandins, prostacyclin, and thromboxanes in the physiologic control of the uterus and in parturition. Seminars in Perinatology 4 45-66.

Reese J, Paria BC, Brown N, Zhao X, Morrow JD \& Dey SK 2000 Coordinated regulation of fetal and maternal prostaglandins directs successful birth and postnatal adaptation in the mouse. PNAS 97 9759-9764.
Riccardi C, Cifone MG \& Migliorati G 1999 Glucocorticoid hormoneinduced modulation of gene expression and regulation of T-cell death: role of GITR and GILZ, two dexamethasone-induced genes. Cell Death and Differentiation $61182-1189$.

Sakkas D, Lu C, Zulfikaroglu E, Neuber E \& Taylor HS 2003 A soluble molecule secreted by human blastocysts modulates regulation of HOXA10 expression in an epithelial endometrial cell line. Fertility and Sterility $801169-1174$.

Salih SM \& Taylor HS 2004 HOXA10 gene expression in human fallopian tube and ectopic pregnancy. American Journal of Obstetrics and Gynecology 190 1404-1406.

Satokata I, Benson G \& Maas R 1995 Sexually dimorphic sterility phenotypes in Hoxa10-deficient mice. Nature 374 460-463.

Scherle PA, Ma W-G, Lim H, Dey SK \& Trzaskos JM 2000 Regulation of cyclooxygenase-2 induction in the mouse uterus during decidualization. An event of early pregnancy. Journal of Biological Chemistry 275 37086-37092.

Simmons DM, Arriza JL \& Swanson LW 1989 A complete protocol for in situ hybridization of messenger RNAs in brain and other tissues with radiolabeled single-stranded RNA probes. Journal of Histotechnology 12 169-181.

Slattery MM \& Morrison JJ 2002 Preterm delivery. Lancet $\mathbf{3 6 0}$ 1489-1497.

Smith R, Mesiano S \& McGrath S 2002 Hormone trajectories leading to human birth. Regulatory Peptides 108 159-164.

Sugimoto Y, Yamasaki A, Segi E, Tsuboi K, Aze Y, Nishimura T, Oida H, Yoshida N, Tanaka T, Katsuyama M et al. 1997 Failure of parturition in mice lacking the prostaglandin $\mathrm{F}$ receptor. Science $277681-683$.

Takanami-Ohnishi Y, Asada S, Tsunoda H, Fukamizu A, Goto K, Yoshikawa H, Kubo T, Sudo T, Kimura S \& Kasuya Y 2001 Possible involvement of p38 mitogen-activated protein kinase in decidual function in parturition. Biochemical and Biophysical Research Communications 288 1155-1161.

Taylor HS, Arici A, Olive D \& Igarashi P 1998 HOXA10 is expressed in response to sex steroids at the time of implantation in the human endometrium. Journal of Clinical Investigation 101 1379-1384.

Taylor HS, Igarashi P, Olive DL \& Arici A 1999a Sex steroids mediate HOXA11 expression in the human peri-implantation endometrium. Journal of Clinical Endocrinology and Metabolism 84 $1129-1135$.

Taylor HS, Bagot C, Kardana A, Olive D \& Arici A 1999b HOX gene expression is altered in the endometrium of women with endometriosis. Human Reproduction 14 1328-1331.

Troy PJ, Daftary GS, Bagot CN \& Taylor HS 2003 Transcriptional repression of peri-implantation EMX2 expression in mammalian reproduction by HOXA10. Molecular and Cellular Biology 23 1-13.

Tsuboi K, Sugimoto Y, Iwane A, Yamamoto K, Yamamoto S \& Ichikawa A 2000 Uterine expression of prostaglandin H2 synthase in late pregnancy and during parturition in prostaglandin $\mathrm{F}$ receptordeficient mice. Endocrinology 141 315-324.

Tsuboi K, Iwane A, Nakazawa S, Sugimoto Y \& Ichikawa A 2003 Role of prostaglandin $\mathrm{H} 2$ synthase 2 in murine parturition: study on ovariectomy-induced parturition in prostaglandin $\mathrm{F}$ receptor-deficient mice. Biology of Reproduction 69 195-201.

Tynan SH, Lundeen SG \& Allan GF 2004 Cell type-specific bidirectional regulation of the glucocorticoid-induced leucine zipper (GILZ) gene by estrogen. Journal of Steroid Biochemistry and Molecular Biology $91225-239$.

Uozumi N, Kume K, Nagase T, Nakatani N, Ishii S, Tashiro F, Komagata Y, Maki K, Ikuta K, Ouchi Y et al. 1997 Role of cytoplasmic phospholipase A2 in allergic response and parturition. Nature 390 618-622.

Vogel P, Magert H-J, Cieslak A, Adermann K \& Forssmann W-G 1996 hDIP - a potential transcriptional regulator related to murine TSC-22 and Drosophila shortsighted (shs) - is expressed in a large number of human tissues. Biochimica et Biophysica Acta 1309 200-204. 
Wang H \& Hirsch E 2003 Bacterially-induced preterm labor and regulation of prostaglandin-metabolizing enzyme expression in mice: the role of Toll-like receptor 4. Biology of Reproduction 69 1957-1963.

Wang LF, Luo HZ, Zhu ZM \& Wang JD 2004 Expression of HOXA11 gene in human endometrium. American Journal of Obstetrics and Gynecology 191 767-772.

Wang Z \& Tai HH 1998 Interleukin-1 beta and dexamethasone regulate gene expression of prostaglandin $\mathrm{H}$ synthase-2 via the NF-kB pathway in human amnion derived WISH cells. Prostaglandins, Leukotrienes, and Essential Fatty Acids 59 63-69.

Watanabe H, Suzuki A, Kobayashi M, Takahashi E, Itamoto M, Lubahn D, Handa H \& Iguchi T 2003 Analysis of temporal changes in the expression of estrogen-regulated genes in the uterus. Journal of Molecular Endocrinology 30 347-358.

Weiss G 2000 Endocrinology of parturition. Journal of Clinical Endocrinology and Metabolism 85 4421-4425.

Received 7 July 2005

First decision 19 August 2005

Revised manuscript received 10 November 2005

Accepted 8 December 2005 